\title{
A Versatile Technique to Produce Porous Polymeric Scaffolds: The Thermally Induced Phase Separation (TIPS) Method
}

\author{
Gioacchino Conoscenti*, Vincenzo La Carrubba and Valerio Brucato
}

Department of Civil, Environmental, Aerospace, Materials Engineering, University of Palermo, Avenue of Sciences, Palermo, Italy

${ }^{*}$ Corresponding author: Gioacchino Conoscenti, Department of Civil, Environmental, Aerospace, Materials Engineering, University of Palermo, Avenue of Sciences - 90128, Palermo, Italy, Tel: +39 0912388 6472; E-mail: gioacchino.conoscenti@unipa.it

Received Date: May 19, 2017; Accepted Date: May 25, 2017; Published Date: June 01, 2017

Copyright: (C) 2017 Conoscenti G, This is an open-access article distributed under the terms of the Creative Commons Attribution License, which permits unrestricted use, distribution, and reproduction in any medium, provided the original author and source are credited.

Citation: Conoscenti G, Carrubba VL, Brucato V. A versatile technique to produce porous polymeric scaffolds: the Thermally Induced Phase Separation (TIPS) method. Arch Chem Res. 2017, 1:2.

\section{Abstract}

Among the various scaffold fabrication techniques, thermally induced phase separation (TIPS) is one of the most versatile methods to produce porous polymeric scaffold and it has been largely used for its capability to produce highly porous and interconnected scaffolds. The scaffold architecture can be closely controlled by varying the process parameters, including polymer type and concentration, solvent/non-solvent ratio and thermal history. TIPS technique has been widely employed, also, to produce scaffolds with a hierarchical pore structure and composite polymeric matrix/inorganic filler foams.

Keywords Porous scaffolds; Thermally induced phase separation; Gradient

\section{Introduction}

The so-called scaffold is one of the building blocks of the tissue engineering approach; the design of a scaffold includes the selection of its constitutive material, its design architecture and often the surface and/or bulk treatment(s) [1]. The scaffold is a three-dimensional support suitable for the growth of a tissue or an organ, aiming to temporarily replace the functions of the extracellular matrix and to guide the proliferation and growth of cells [2]. Several studies showed that, without a proper template, the cells tend to arrange in a two-dimensional (2D) layer forming a flat structure with poor mechanical properties [3]. In particular, by using scaffolds, transplanted cells can be delivered to a specific site place in a tissue hence driving the growth of cells inside towards a desired target. Thus, scaffolds represent the free space available for the tissue to develop and a physical support for cell growth. To achieve these objectives, the scaffold must possess some basic requirements: biocompatibility, biodegradability, topological features, mechanical properties, sterilazibility, etc. [4]. Several biomaterials (natural $[5,6]$ or synthetic $[7,8]$ ) have been exploited to mimic as good as possible the tissue to regenerate. The choice of the biomaterial is also related to its production technique, in order to attain the optimum porosity, pore interconnectivity and mechanical properties. Among the possible techniques the most widely adopted are solvent-casting and particulate leaching [9], phase separation methodologies [10], gas-foaming [11], CAD/CAM based methods [12] and electrospinning [13].

\section{Thermally Induced Phase Separation (TIPS) Technique}

Thermally Induced Phase Separation (TIPS) is a widely adopted procedure for scaffold preparation, suitable for obtaining a well-interconnected porous structure.

This technique is based on the change in temperature to induce the de-mixing of a homogeneous polymer solution, thus creating a multi-phase system. When the de-mixing of the solution occurs, the homogeneous solution separates into a polymer-rich and a polymer-less phase. The de-mixing can be solid-liquid (usually for binary polymer-solvent mixtures), or liquid-liquid (usually for ternary polymer/solvent/non-solvent mixtures).

One of the most relevant advantages of this technique is the possibility to obtain a well-interconnected polymer network with an easy-to-tune, fast and adaptable process. As a function of the separations conditions, it is possible to obtain a wide range of different morphologies with various characteristics, such as: open or close pores, fibrous structure, membrane/like architecture, etc. [14-17].

Lots of polymers have been used, among which it is worth to mention polylactic acid[18], polyurethane[19], polycaprolacton [20], etc.

Figure 1 reports the phase diagram of a typical ternary solution. The thermodynamic equilibrium boundaries are represented by the binodal and the spinodal curve. At high temperatures (above the binodal curve) the polymer solution is in the one-phase region and the solution is homogeneous. Upon decreasing the temperature, the solution reaches the binodal curve and de-mixing into a polymer-rich and a polymer-lean phase occurs. The area between binodal and spinodal curve is 
the metastable region, where a (quite slow) mechanism of nucleation and growth takes place, leading to (relatively) large pores. On the opposite, the separation mechanism taking place in the spinodal region, called spinodal decomposition, is very fast and usually leads to the formation of a network of small interconnected pores.

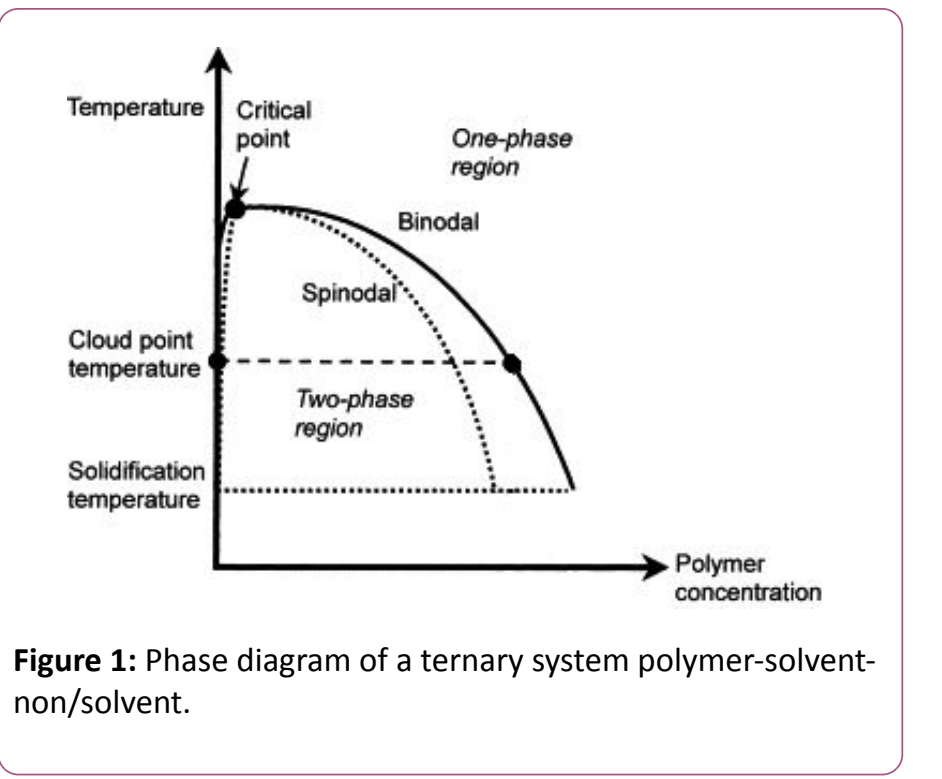

\section{Operating Parameters}

Distribution, shape and size of the pores are the result of a delicate balance between different parameters among which the most important are polymer type and concentration, polymer molecular weight, solvent/non-solvent ratio and cooling path.

- Polymer concentration: as the concentration increases, keeping constant the cooling path, pore dimension and porosity decrease accordingly.

- Polymer molecular weight: an increase of the molecular weight implies an increase of the viscosity of the solution that plays a crucial role during the formation of the two phases. The foams at higher molecular weight result more homogeneous compared to the others ones.

- Solvent/non-solvent ratio: the first attempts to produce scaffold via TIPS were carried out starting from a binary solution polymer/solvent. In this case, the scaffolds present a ladder-like structure due to the solidification of the solvent, and the final scaffold structure is mainly dominated by the progress of the crystallization of the solvent. Inducing a liquid-liquid phase separation by adding a non-solvent allowed a significant improvement in the scaffold architecture together with an increase in the sensitivity of the solution to the tuneable parameters, above all the thermal history

- Cooling path: one of the principal advantages of the TIPS technique is the possibility to tune the structure by playing with the temperature vs. time pathway. As a matter of fact, starting from the same solution, the final morphology of the scaffold can exhibit very different features (in terms of pore dimension, distribution, interconnectivity, etc) by simply tuning the cooling path. The graph in Figure 2 [21] reports the trend of pore dimension as a function of the temperature and time. It is evident that at $25^{\circ} \mathrm{C}$ pore size is basically independent of the demixing time. For the examined solution at $25^{\circ} \mathrm{C}$ the predominant process is spinodal decomposition, not related to time. For the remaining two temperatures, $30^{\circ} \mathrm{C}$ and $35^{\circ} \mathrm{C}$, when increasing the demixing time from 15 to 45 minutes the pore dimensions are more than quintupled, and the sigmoidal shape typically exhibited by saturation or "space-filling" processes (like nucleation and growth) is visible.

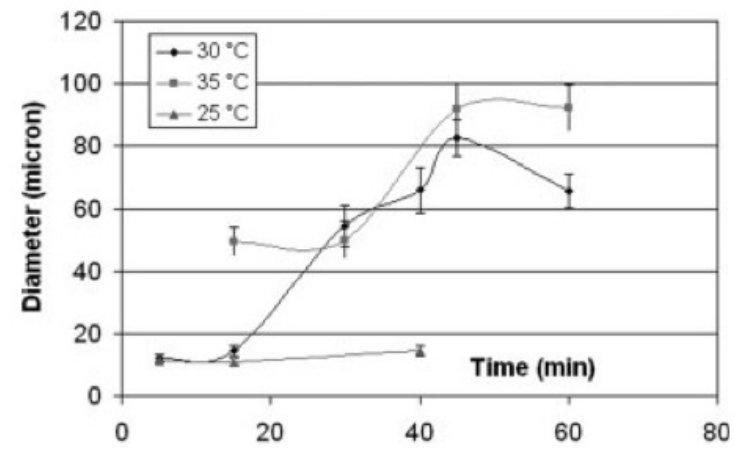

Figure 2: Effect of time and temperature in pore dimension.

These criteria have a significant influence not only on the onset of the phase separation, in which the domains of the nucleating phase start to grow, but also in the next steps. Indeed, the coalescence of the separate phase, continues minimising the free energy due to the presence of the interfacial surfaces. This process, called coarsening, causes the increase of the pore dimension [22].

By taking advantage of the TIPS process, since the pore dimension is mainly dependent on the residence time in the metastable region [23], Mannella et al. [24] proposed an experimental apparatus able to impose a different $T$ vs. time pathway on two sides of a sample. In this way a gradient of pore dimension was obtained along the scaffold thickness in a single step, without requiring the junction of the multiple layers.

TIPS technique was adopted, too, to produce also composite scaffold by adding ceramic fillers: hydroxyapatite, bioglass, keratin [25], etc. starting from binary $[26,27]$ or ternary solution [28].

\section{Conclusion}

TIPS technique allows the formation of an interconnected porous structure through an easy-to-tune fabrication process. The technique has demonstrated a remarkable potential to obtain scaffolds with a wide range of pore sizes. The process can be tuned by manipulating the processing parameters, e.g. thermal history, to fabricate scaffolds with the desired architectures and pore morphologies. By taking advantage of the relative ease in tuning the final topology of the scaffold, foams with hierarchical structures have been obtained by creating thermal or concentration gradient. Furthermore, inorganic filler 
can be suitably incorporated into the scaffold matrix in a onestep process to enhance the bioactivity and the mechanical properties of the scaffold.

\section{References}

1. Li J, Connell S, Shi R (2010) Biomimetic architectures for tissue engineering. Biomim Archit Tissue Eng 487-507.

2. Tang D, Tare RS, Yang LY, Williams DF, Ou KL, et al. (2016) Biofabrication of bone tissue: Approaches, challenges and translation for bone regeneration. Biomaterials 83: 363-382.

3. Tian XF, Heng BC, Ge Z, Lu K, Rufaihah AJ, et al. (2008) Comparison of osteogenesis of human embryonic stem cells within 2D and 3D culture systems. Scand J Clin Lab Invest 68: 58-67.

4. Seol YJJ, Park DY, Park JY, Kim SW, Park SJ, et al. (2013) A new method of fabricating robust freeform 3D ceramic scaffolds for bone tissue regeneration. Biotechnol Bioeng 110: 1444-1455.

5. Andersen T, Auk-Emblem P, Dornish M (2015) 3D cell culture in alginate hydrogels. Microarrays 4: 133-161.

6. Mullen LM, Best SM, Ghose S, Wardale J, Rushton N, et al. (2015) Bioactive IGF-1 release from collagen-GAG scaffold to enhance cartilage repair in vitro. J Mater Sci Mater Med 26: 1-8.

7. Curran JM, Fawcett S, Hamilton L, Rhodes NP, Rahman CV, et al. (2013) The osteogenic response of mesenchymal stem cells to an injectable PLGA bone regeneration system. Biomaterials 34 : 9352-9364.

8. Du Y, Chen X, Hag Koh Y, Lei B (2014) Facilely fabricating PCL nanofibrous scaffolds with hierarchical pore structure for tissue engineering. Mater Lett 122: 62-65.

9. Huang R, Zhu X, Zhao T, Wan A (2014) Preparation of tissue engineering porous scaffold with poly (lactic acid) and polyethylene glycol solution blend by solvent-casting/particulateleaching. Mater Res Express 1: 45403.

10. Hsu SH, Huang S, Wang YC, Kuo YC (2013) Novel nanostructured biodegradable polymer matrices fabricated by phase separation techniques for tissue regeneration. Acta Biomater 9: 6915-6927.

11. Goswami J, Bhatnagar N, Mohanty S, Ghosh AK (2013) Processing and characterization of poly (lactic acid) based bioactive composites for biomedical scaffold application. Express Polym Lett 7: 767-777.

12. Lee JW, Kim JY, Cho DW (2010) Solid free-form fabrication technology and its application to bone tissue engineering. Int J Stem Cells 3: 85-95.

13. Song X, Wei L, Chen A, Shao Y (2015) Poly(L-lactide) nanofibers containing trypsin for gelatin digestion. Fibers Polym 16: 867-874.

14. He L, Zhang Y, Zeng X, Quan D, Liao S, Y. Zeng, et al. (2009) Fabrication and characterization of poly(I-lactic acid) 3D nanofibrous scaffolds with controlled architecture by liquid-liquid phase separation from a ternary polymer-solvent system. Polymer (Guildf) 50: 4128-4138.
15. Kim JW, Taki K, Nagamine S, Ohshima M (2009) Preparation of porous poly (L-lactic acid) honeycomb monolith structure by phase separation and unidirectional freezing. Langmuir 25: 5304-5312.

16. Phaechamud T, Chitrattha S (2016) Pore formation mechanism of porous poly (dl-lactic acid) matrix membrane. Mater Sci Eng C 61: 744-752.

17. Yang F, Murugan R, Ramakrishna S, Wang X, Ma WY, et al. (2004) Fabrication of nano-structured porous PLLA scaffold intended for nerve tissue engineering. Biomaterials 25: 1891-1900.

18. Ruggiero F, Netti PA, Torino E (2015) Experimental investigation and thermodynamic assessment of phase equilibria in the PLLA/ Dioxane/water ternary system for applications in the biomedical field. Langmuir 31: 13003-13010.

19. Jing X, Mi HY, Salick MR, Cordie T, Crone WC, et al. (2014) Morphology, mechanical properties, and shape memory effects of poly (lactic acid)/thermoplastic polyurethane blend scaffolds prepared by thermally induced phase separation. J Cell Plast 50: 361-379.

20. Salerno A, Domingo C (2015) Pore structure properties of scaffolds constituted by aggregated microparticles of PCL and PCL-HA processed by phase separation. J Porous Mater 22: 425-435.

21. Carfi Pavia F, La Carrubba V, Piccarolo S, Brucato V (2008) Polymeric scaffolds prepared via thermally induced phase separation: Tuning of structure and morphology. J Biomed Mater Res-Part A 86: 459-466.

22. Li D, Krantz WB, Greenberg AR, Sani RL (2006) Membrane formation via thermally induced phase separation (TIPS): Model development and validation. J Memb Sci 279: 50-60.

23. Mannella GA, Carfi Pavia F, Conoscenti G, La Carrubba V, Brucato V (2014) Evidence of mechanisms occurring in thermally induced phase separation of polymeric systems. J Polym Sci Part B Polym Phys 52: 979-983.

24. Mannella GA, Conoscenti G, Carfi Pavia F, La Carrubba V, Brucato V (2015) Preparation of polymeric foams with a pore size gradient via thermally induced phase separation (TIPS). Mater Lett 160: $1-4$

25. Puglia D, Ceccolini R, Fortunati E, Armentano I, Morena F, S. Martino, et al. (2015) Effect of processing techniques on the 3D microstructure of poly (L-lactic acid) scaffolds reinforced with wool keratin from different sources. J Appl Polym Sci 132.

26. Maquet V, Boccaccini AR, Pravata L, Notingher I, Jérôme R (2003) Preparation, characterization, and in vitro degradation of bioresorbable and bioactive composites based on Bioglass(R)filled polylactide foams. Biomed Mater Res A 66:335-346.

27. Wang X, Song G, Lou T (2010) Fabrication and characterization of nano composite scaffold of poly (L-lactic acid)/hydroxyapatite. J Mater Sci Mater Med 21: 183-188.

28. Ghersi G, Carfi Pavia F, Conoscenti G, Mannella GA, Greco S, et al. (2016) PLLA scaffold via TIPS for bone tissue engineering. Chem Eng Trans 49: 301-306. 